\title{
El e-book científico-técnico en el mercado español a partir del análisis de las agencias de suscripciones
}

\author{
Por Irene-Sofía Romero-Otero, Elea Giménez-Toledo y Juan-Carlos Martín-González
}

\begin{abstract}
Resumen: Se analizan la gestión, las estrategias comerciales, el marketing y los usuarios del libro electrónico científicotécnico en el mercado español desde el punto de vista de las principales agencias de suscripciones. Los responsables de Ebsco y Swets en España fueron entrevistados con el fin de obtener el panorama general presentado. La opinión más extendida en el contexto académico -investigadores, bibliotecarios, editores y agencias de suscripciones- es que la aceptación del libro electrónico en el ámbito académico es mucho más lenta en comparación con las revistas electrónicas, pero cada vez es más importante.

Palabras clave: Libro electrónico, Agencias de suscripciones, Oferta de libros electrónicos, Mercado español.
\end{abstract}

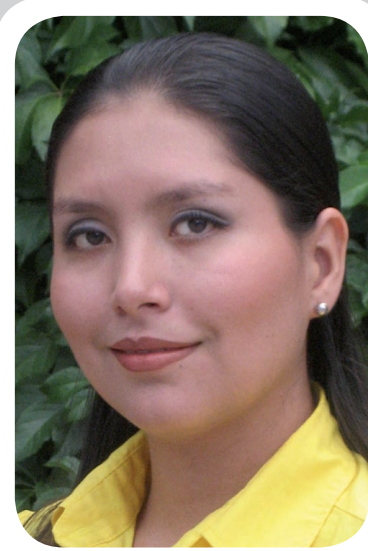

Irene-Sofía Romero-Otero, licenciada en comunicación social y periodismo por la Universidad Surcolombiana de Colombia, es investigadora predoctoral del Programa Junta para la Ampliación de Estudios (JAE) del Csic y miembro del grupo de investigación de evaluación de publicaciones científicas del Instituto de Estudios Documentales sobre Ciencia y Tecnología (ledcyt).

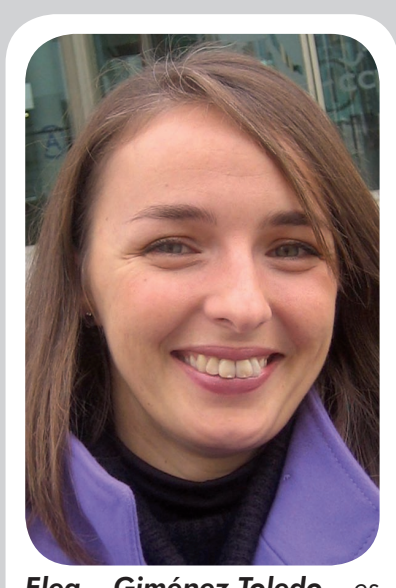

Elea Giménez-Toledo es doctora en documentación por la Universidad Carlos III de Madrid. Es científica titular del Consejo Superior de Investigaciones Científicas y miembro del grupo de investigación de evaluación de publicaciones científicas del Instituto de Estudios Documentales sobre Ciencia y Tecnología (ledcyt).

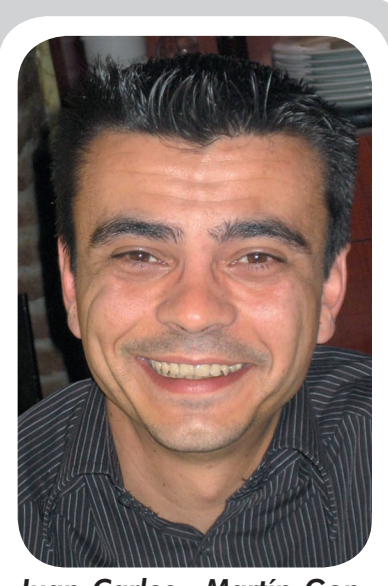

Juan-Carlos Martín-González, licenciado en documentación por la Universidad de Salamanca, es Country Manager de Swets en España. Ha publicado artículos y participado en congresos con trabajos sobre aspectos comerciales de las revistas electrónicas y la industria editorial, información científicotécnica y perfiles profesionales en documentación.

\section{Title: The scientific-technical e-book in the Spanish market: an overview from the subscription} agent perspective

Abstract: Management, trade strategies, marketing and users of scientific-technical e-books in the Spanish market are analysed in this paper, from the point of view of the main subscription agents. Swets and Ebsco country managers in Spain were interviewed to obtain a general overview. The most widely held opinion in the scholarly context-researchers, librarians, publishers and subscription agents-is that the scholarly e-book is being accepted much more slowly than e-journals but will become more and more important.

Keywords: E-book, Subscription agents, E-books supply, Spanish market.

Romero-Otero, Irene-Sofía; Giménez-Toledo, Elea; Martín-González, Juan-Carlos. "El e-book científico-técnico en el mercado español a partir del análisis de las agencias de suscripciones". El profesional de la información, 2010, enerofebrero, v. 19, n. 1, pp. 21-27.

DOI: 10.3145/epi.2010.ene.03

\section{Introducción}

Aunque el libro electrónico (en adelante e-book) lleva presente poco tiempo en el mercado español, hay que reconocer los esfuerzos que vienen realizando tanto las editoriales como las agencias de suscripciones para contribuir de alguna manera a su proceso de consolidación.
Resulta interesante el punto de vista de estas agencias, gracias a la enorme experiencia acumulada con la gestión de las colecciones de revistas electrónicas y al profundo conocimiento que tienen de los demandantes de información científica. Tales agencias han considerado que el siguiente paso lógico era hacer una apuesta fuerte por la comercialización del $e$-book en el mercado español. Por ello están desempeñando un papel cla- 
ve como intermediadoras en su adquisición, gestión y venta. Las tareas que ya asumían con las bibliotecas al gestionar las suscripciones de publicaciones periódicas -selección, negociación con editores, gestión de pagos, incidencias, suscripciones, asesoramiento, facturaciones, renovaciones- son realizadas también con el $e$-book, aunque con las particularidades que éste presenta.

La mayoría de bibliotecas o instituciones interesadas en adquirir colecciones de $e$-books opta por realizar su compra a través de las agencias de suscripciones por las facilidades que brindan, desde el momento en que centralizan el acceso y muchos aspectos de la gestión por medio de un único punto de contacto (Collison, 2006). El principal valor añadido que ofrecen las agencias es la eficiente administración, porque ayudan a ambas partes (editor y cliente) a reducir costos y mejorar los servicios, contribuyendo de esta manera a flexibilizar los procesos de negociación (Harwood, 2003).

A pesar de lo mediático que ha sido el e-book a lo largo de 2009, la impresión más extendida en el entorno académico -investigadores, bibliotecarios, editores y las propias agencias de suscripción- es que el $e$-book de carácter científico está teniendo una aceptación mucho más lenta que su antecedente más inmediato, la revista electrónica.

En este sentido, este trabajo pretende profundizar en el proceso de expansión y aceptación del $e$-book en el entorno científico, eligiendo para ello la perspectiva de las agencias de suscripciones como uno de los actores protagonistas de este escenario.

\section{"Las agencias de suscripciones están desempeñando un papel destacado en la adquisición, gestión y venta de e-books"}

\section{Objetivos}

Este artículo pretende analizar la gestión, oferta y estrategia comercial del $e$-book por parte de las principales agencias de suscripciones presentes en España, Ebsco y Swets, poniendo especial atención en el ámbito de las ciencias sociales y las humanidades, áreas en las que la monografía ha sido siempre protagonista.

Los tres aspectos esenciales que se abordarán son:

a) Estrategia comercial, con la intención de:

- conocer sus inicios y evolución en el mercado español desde la perspectiva de las agencias de suscripciones y la visión que tienen sobre el futuro del mismo;
- describir los modelos de negocio predominantes para su comercialización; y

- explorar las colecciones de e-books en ciencias sociales y humanidades.

b) Usuarios o instituciones que más demandan colecciones de $e$-books a estas agencias en España, así como las características de las licencias que plantean los editores.

c) Modelo de marketing para la venta de las colecciones de $e$-books que emplean las agencias de suscripciones.

\section{Metodología}

Este estudio se basa en sendas entrevistas estructuradas realizadas a responsables en España de las dos agencias de suscripciones. La entrevista está organizada en tres bloques, que se corresponden con áreas de interés señaladas en los objetivos. Las entrevistas tuvieron una duración de más de una hora y fueron realizadas a Juan-Carlos Martín-González, director de Swets en España (y coautor de este artículo) el 4 de marzo de 2009 y a Jesús Muñoz, director del Departamento de Recursos Electrónicos de Ebsco en España el 20 de enero de 2009.

Por otra parte, la revisión bibliográfica, así como la documentación proporcionada por las agencias de suscripciones y obtenida a partir de sus sitios web, han permitido completar y contextualizar el panorama del $e$-book en el mercado español.

\section{"El e-book científico está teniendo una aceptación más lenta que su antecedente más inmediato, la revista electrónica"}

\subsection{Estrategia comercial}

\section{Mercado del $e$-book en España}

La comercialización de los e-books se inició en España en 2005, según Juan-Carlos Martín-González: "fue en 2005 cuando se dieron las primeras iniciativas por parte del sector editorial", y a su vez Jesús Muñoz añadió que "fue el momento en el que, de alguna manera, el mercado del $e$-book empezó a tomar forma y a tener una cierta demanda".

La visión que tienen estas agencias sobre el papel que han desempeñado los editores frente a los $e$-books es positiva. Así lo deja ver el representante de Swets: 
"los editores están apostando fuerte, porque ya han llegado a una especie de techo en cuanto a la comercialización de revistas en formato digital y el paso lógico es ampliar ese modelo a monografías (...). Se está viendo que aparte del esfuerzo de infraestructuras y de proyectos técnicos de desarrollo de las colecciones, también está habiendo un esfuerzo comercial importante".

A pesar de ello, el mercado del e-book en España durante estos cuatro años ha sido lento, no ha respondido como se esperaba. Según el representante de Ebsco "se trata de un mercado muy reciente, aún no está maduro y es todavía muy limitado. Si bien la transición de las revistas electrónicas se hizo en unos cinco o seis años, la del e-book está tardando un poco más". Para dicha agencia es probable que su tardanza se deba a aspectos como el desconocimiento de las ventajas que ofrece este soporte, su limitación a un sector de la comunidad académica restringido, ya que la mayor parte del alumnado no lo emplea por desconocimiento del inglés -idioma en el que se publican la mayor parte de los contenidos científicos-, y porque el sistema académico español de momento sigue siendo muy tradicional y el uso que hace el estudiante en los primeros años de esos medios de información es muy limitado.

Respecto al idioma como posible inconveniente, es importante señalar que el hecho de que la oferta del $e$ book sea predominantemente en inglés no tendría por qué suponer un freno en su utilización, puesto que ocurre lo mismo con las revistas electrónicas y, sin embargo, se han consolidado perfectamente.

Los obstáculos que señala el representante de Ebsco, de una manera u otra concuerdan con la reflexión que ha hecho Cuesta (2005) sobre las razones que han limitado el éxito de los e-books en el mercado español: su alto precio, la escasa oferta de catálogos, el predominio del inglés y las excesivas medidas de protección establecidas por la industria para luchar contra la piratería han hecho de los e-books soportes demasiado personales, es decir, no pueden ser prestables a otras personas, por tener muchas medidas de seguridad.

En lo que concierne al precio habría que destacar que, a día de hoy, el representante de Swets considera que "un e-book puede ser incluso más barato que su equivalente impreso. Solamente las grandes colecciones pueden ser consideradas 'caras' por la falta de relevancia de muchos de sus contenidos, la selección individual de títulos puede suponer un ahorro importante en el presupuesto de monografías".

Pese a las anteriores limitaciones, las agencias de suscripciones son optimistas sobre el futuro del $e$-book en el contexto español, porque creen que este formato se consolidará, pero que irá a su propio ritmo, y así lo señala el representante de Ebsco: "se consolidará como la revista electrónica, pero el salto en el entorno académico en relación con el e-book dentro del aula, más bien va a depender de los cambios que tenga el sistema educativo que del propio producto". Es probable que la progresiva adaptación al proceso de Bolonia, uno de cuyos pilares es la autonomía en el aprendizaje y en la investigación por parte del alumno, potencie un mayor y más variado uso de las fuentes de información, lo que vendría a reforzar el rol del e-book. Por su parte, el representante de Swets opina que "es un formato que crecerá y que formará parte de la vida académica y científica en un período breve de tiempo; probablemente en unos tres o cinco años ya tenga una presencia bastante importante".

\section{"Las agencias creen que este formato crecerá y formará parte de la vida académica y científica en un período corto"}

En este punto vale la pena mencionar que la estrategia que vienen empleando tanto los editores como las agencias de suscripciones es una apuesta por la integración. Esto supone ubicar los e-books en las plataformas que ya están destinadas para otro tipo de productos digitales en las bibliotecas; en pocas palabras, se trata "de la capacidad de tener en un click una multitud de materiales que anteriormente estaban diseminados" (Ebsco).

El volumen de la edición de $e$-books puede medirse a través de las cifras sobre su comercialización en el mercado español (sólo corresponden al mercado interno):

- En 2005 "la facturación de los e-books fue muy reducida: 27,3 millones de euros, lo que representa el 9,5\% de la facturación total de la edición en otros soportes diferentes del papel. El peso de la facturación de los e-books ocupa el cuarto lugar en el total de la facturación de otros soportes" (Larrañaga-Rubio, 2008).

- En 2006 la facturación fue de 31,73 millones de euros, lo que representa el $9,5 \%$ de la facturación total de la edición en otros soportes diferentes del papel $(\mathrm{Fe}$ deración de Gremios de Editores de España, 2006).

- Según datos de la FGEE, en 2007 el 10,5\% de la facturación editorial anual responde a la edición digital (Federación de Gremios de Editores de España, 2007) ${ }^{1}$.

Ante este panorama queda claro que la presencia de los e-books en el mercado español es escasa, 
tal como escribe Larrañaga: "Los e-books tienen un peso muy pequeño en la panorámica del mundo de la edición en España... la producción de $e$-books es muy reducida en nuestro mercado editorial" (LarrañagaRubio, 2008).

\section{"Los clientes que más demandan e- books en España son las instituciones académicas, bibliotecas universitarias y centros de investigación de titularidad estatal"}

La opinión que tiene el sector editorial se refleja en el estudio "Digitalización del libro en España" (Ediciona; Dosdoce.com, 2008), donde se dice que el 57\% de los profesionales del sector cree que ambos soportes (papel y electrónico) convivirán. Sólo un $15 \%$ opinó que los e-books llegarán a imponerse sobre los libros impresos.

Sin embargo parece que será cuestión de tiempo que logre posicionarse mejor y así lo dejan ver las conclusiones a las que llegaron varios expertos y profesionales del sector editorial reunidos en el foro de Publidisa 2009: es posible que en 2010 el $e$-book (entendido en sentido estricto, no referido a cualquier formato digital) suponga un 5\% del total del negocio editorial, un porcentaje que se duplicará para 2013, hasta lograr en 2015 el 50\% del negocio editorial (eForo Publidisa, $2009)^{2}$.

En cuanto al $e$-book en el ámbito académico se puede apreciar que "el aumento de las monografías electrónicas entre los miembros de Rebiun ha experimentado un crecimiento del $37 \%$ entre 2005 y 2007, pasando de 2.700.000 a 3.695.000 e-libros accesibles por pago" (Martín-González; Pivetta, 2008). Esto refleja que el e-book va teniendo aceptación por parte de investigadores, profesores y estudiantes.

Estos datos parecen lejos de las previsiones que el sector editorial a escala mundial hace sobre el peso del e-book en el mercado, pues en la Feria del libro de Frankfurt de 2007, el 44\% de profesionales (1.324 de 86 países) respondieron que el $e$-book será una parte del sector editorial que experimentará un crecimiento destacado (Rodríguez, 2007) y un año más tarde, en 2008 en la misma feria, las predicciones apuntaban a que en 2018 el volumen de negocio del $e$-book superará al del libro impreso (Díaz-Noci, 2009). A su vez, en la feria anual Digital Book 2008, patrocinada por International Digital Publishing Forum, los presentadores indicaban que "los editores ven un rápido crecimiento en el mercado del libro digital y que el formato se está convirtiendo en una parte cada vez más importante de su negocio" (Teicher, 2008). Estas visiones reflejan la actitud optimista que hay en el sector editorial a escala mundial con respecto al futuro del libro electrónico.

\section{Modelos de negocio}

Las agencias de suscripciones respetan los modelos de negocio u opciones de compra que plantea cada editor y los emplean en sus negociaciones con las bibliotecas. No obstante, dentro de sus funciones está también ayudar a flexibilizar la postura de los distintos actores, para poder llegar a acuerdos con ambas partes (cliente y editorial). Es por esto que según el representante de Swets "las editoriales plantean un modelo, pero la personalización es necesaria, por ello nosotros también jugamos ahí un papel importante".

\section{"Los cuatro años de trayectoria del e- book en España no han sido suficientes para lograr su consolidación"}

Las opciones de compra se refieren por una parte al modo de adquisición y de pago de $e$-books y por otra a la estructura de los contenidos. Las opciones más comunes de adquisición y pago entre los editores y, por tanto, las que ofrecen las agencias de suscripciones son: la compra única, mediante la cual se garantizan unos derechos de archivo que en principio tienden a ser perpetuos, es decir, "la institución adquiere en propiedad el contenido seleccionado, manteniendo los derechos de acceso sobre el mismo de forma indefinida"; y la suscripción, "por la que la institución obtiene derechos sobre el material contratado y sus actualizaciones, o incluso en algunos casos sobre nuevas ediciones y nuevas obras añadidas a colecciones, durante un período concreto de tiempo y contra el pago de una cuota anual" (Martín-González; Pivetta, 2008).

En cuanto al contenido lo más habitual es que se ofrezcan colecciones completas del catálogo o una colección temática. La selección individualizada de títulos sólo se da en algunos casos cuando las editoriales lo permiten, por lo que no es una fórmula habitual de compra.

Sin embargo es interesante la observación del representante de Swets sobre estas opciones de compra: "una de las tendencias que se ha visto durante estos cuatro años es que se intenta extrapolar el modelo de comercialización de las revistas electrónicas al e-book; 
el concepto del big deal, tan de moda hace algunos años, se ha intentado aplicar también a los e-books y en muchos casos se ha visto que el interés por parte de la comunidad profesional no es el mismo. Este modelo no es aplicable a los $e$-books porque la manera de adquirirlos, seleccionarlos y de desarrollar una colección es completamente diferente a los de las revistas electrónicas. En este sentido lo que busca Swets es flexibilizar los modelos para que la manera de adquirir el $e$-book se ajuste más a la realidad y a los procesos actuales de adquisición de monografías dentro de las bibliotecas científicas y universitarias principalmente".

Siguiendo con el caso de Swets, su representante afirma que "la fórmula de venta predominante ha sido por colecciones temáticas, aunque en 2008 se incrementó la de selección título a título". Por su parte el representante de Ebsco indica: "sus editores están más interesados en la colección que en el libro individual, porque luego la gestión técnica que tienen que hacer ellos es mucho más flexible con una colección cerrada que con títulos individuales". Sin embargo ambos representantes coinciden en que algunos editores han flexibilizado un poco dichos modelos.

\section{Colecciones de $\boldsymbol{e}$-books en ciencias sociales y huma- nidades en ambas agencias de suscripciones}

La presencia de títulos de e-books en ciencias sociales y humanidades en las editoriales que tienen en común tanto Swets como Ebsco, es menor en comparación con el número de títulos que hay de las ciencias exactas y naturales. Según sus representantes se debe a que:

- Las editoriales tienen una oferta mayor de $e$-books para las disciplinas con más demanda.

- Los usuarios de humanidades y ciencias sociales son más conservadores, lo que ocasiona en ellos mayores recelos en relación al e-book. Sin embargo, según el representante de Ebsco últimamente se percibe un mayor interés por tratar de incorporar este formato al entorno académico.

- Los investigadores de ciencias exactas han aceptado algo más rápido al e-book en comparación con humanidades y ciencias sociales.

\subsection{Usuarios}

Según los representantes de estas agencias de suscripciones, los clientes que más han demandando colecciones de $e$-books en España son principalmente las instituciones académicas, bibliotecas universitarias y centros de investigación de titularidad estatal, como cabría esperar. No obstante, en la mayoría de ocasiones dichas bibliotecas destinan un porcentaje menor a los $e$-books dentro del presupuesto de monografías y esto puede deberse posiblemente a que el libro electrónico aún no se ha consolidado y empieza a ser aceptado lentamente entre los usuarios.

Las licencias establecen los términos en los que los lectores pueden consultar y descargar los e-books. Algunas de las posibilidades que ofrecen son comunes a las revistas electrónicas:

- La licencia puede limitar el acceso a un número concreto de usuarios simultáneos.

- Posibilidad de descarga e impresión con algunas variantes: en algunos casos hay limitaciones de descargas, en otros puede variar por número de páginas, por capítulos, por obra completa, etc.

- Se pueden establecer restricciones en cuanto a los derechos de acceso a lo largo del tiempo.

- Tiende a aplicarse la jurisdicción propia del productor de la obra.

- Se aseguran que los e-books tengan un uso autorizado.

- La institución firmante de la licencia tiene que garantizar un uso apropiado de los $e$-books.

\subsection{Marketing}

Las agencias realizan tareas de marketing, al igual que con el resto de productos de información que comercializan. Para promocionar los e-books, por ejemplo, Ebsco diseña material propio en castellano, de forma que llegue mejor a los clientes y que se conozca la oferta del editor más fácilmente. Swets busca facilitar la tarea del bibliotecario ofreciéndole trials para la valoración previa, aportando material de referencia, manuales de uso, etc.; de esta forma pueden comprobar la utilidad, el interés que puede tener para sus usuarios, la facilidad de uso, etc., y valorar si les interesa comprar o no el e-book o la colección.

\section{"En el mercado editorial español hay una menor oferta de e-books en ciencias sociales y humanas"}

Por otra parte las agencias ofrecen diferentes opciones de precios para las colecciones. Estas variaciones, que en ocasiones constituyen ofertas promocionales y con ellas se consigue atraer algo más al cliente, dependen del tamaño de la biblioteca que está interesada en gestionar la compra, los años de la edición que se desea comprar, el tamaño de la colección temática y el prestigio y reconocimiento de la colección en el ámbito científico. 


\section{Discusión y conclusiones}

A raíz de los resultados obtenidos se llegó a las siguientes conclusiones:

- Los cuatro años de trayectoria del e-book en España no han sido suficientes para lograr su consolidación. Según las agencias de suscripciones analizadas, hoy en día dicho mercado se encuentra limitado, pero va madurando paulatinamente.

- El proceso de transición del e-book en España está siendo algo lento en relación con el que vivieron en su momento las revistas electrónicas, aunque la comparación entre ambos no es del todo adecuada pues cada uno presenta particularidades destacadas. Si bien se ha aceptado el hecho de leer las veinte páginas de un artículo en pantalla o de imprimirlo porque tiene una extensión limitada, parece que existe un cierto recelo a abandonar el libro como objeto, que permite hacer anotaciones, ir de un lado a otro, subrayar, etc. Trasladar el comportamiento y la admiración que se siente hacia el libro impreso al $e$-book supone un salto cultural que implica necesariamente un tiempo mayor de adaptación.

- Las agencias de suscripciones analizadas son optimistas frente al futuro del $e$-book en el mercado español; consideran que finalmente logrará consolidarse y tendrá éxito especialmente en el entorno académico.

- Los editores también están haciendo una apuesta fuerte, lo que se refleja en una oferta amplia y variada de diferentes colecciones temáticas de $e$-books. Los bibliotecarios por su parte deben compaginar en este momento la compra tradicional de monografías, que es lo que realmente están demandando sus usuarios, con las pruebas y pequeñas compras de e-books, que les permitirán irse adentrando en este campo, además de ir conociendo el modelo de negocio e ir familiarizando a sus usuarios con el nuevo soporte.

- En el mercado actual hay variados modelos de adquisición y condiciones de licencias diferentes propuestas por varios editores, lo que genera confusión en las bibliotecas en el momento de gestionar su compra de $e$-books.

- En el mercado editorial español hay una menor oferta de $e$-books en ciencias sociales y humanas; son pocas las editoriales asociadas a agencias de suscripciones que ofrecen este tipo de colecciones, lo que por una parte llama la atención dado el protagonismo del libro en estas áreas, y por otra puede entenderse por el mayor apego al libro como objeto que tienen los humanistas y científicos sociales.

- En cuanto a la disposición de los recursos en los sitios web de las bibliotecas, la estrategia que más frecuentemente están implementando tanto editores como agencias de suscripciones, es la integración del e-book dentro de la plataforma en la que están incorporados los demás recursos electrónicos, con el fin de que el usuario tenga a mano toda la información necesaria presentada en diferentes formatos metabuscables entre sí. Sin embargo hay que considerar que aunque la estrategia es interesante, parece que en un primer momento la oferta de $e$-books en una biblioteca debería aparecer destacada, independientemente de los otros recursos electrónicos, logrando de esta forma que el usuario y visitante de la web de la biblioteca identifique claramente la sección destinada para los e-books como algo novedoso para explorar. Esta opción no implica que internamente la identificación bibliográfica de los e-books y su alojamiento estén integradas con el resto de productos electrónicos, sino más bien que el usuario sepa que hay un enlace dedicado exclusivamente a $e$-books.

\section{Notas}

1. Los anteriores datos se refieren a porcentajes calculados sobre la facturación de lo que Larrañaga-Rubio considera como e-books (online), dentro del porcentaje que suponen los libros que han sido editados en soportes diferentes al papel (cd, dvd, audiolibros, etc.). Igualmente, hay que mencionar que en dicho trabajo los e-books no fueron diferenciados por materias (ficción, científicos, técnicos, etc.).

2. Las cifras correspondientes a los años 2010, 2013 y 2015, se refieren a porcentajes calculados sobre el total del negocio editorial del libro, el cual incluye tanto el papel como el resto de los soportes.

\section{Agradecimientos}

Este trabajo se ha llevado a cabo gracias a la concesión de una beca/contrato predoctoral en el marco del Programa Junta para la Ampliación de Estudios (JAE) del Consejo Superior de Investigaciones Científicas.

\section{Referencias}

Collison, Nick. "On information professionals and subscription agents". Information Outlook, oct 2006.222

http://findarticles.com/p/articles/mi_mOFWE/is_10_10/ai_n27063840/

Cuesta, Albert. "e-Libros". La vanguardia.es, 19 enero 2005.

http://www.lavanguardia.es/premium/publica/publica? COMPID $=5124382$ 0589\&ID_PAGINA $=22780 \& I D \_F O R M A T O=9$

Díaz-Noci, Javier. “2018: ¿Diarios en dispositivos móviles? Libro electrónico, tinta electrónica y convergencia de la prensa impresa y digital". $E l$ profesional de la información, 2009, mayo-junio, v. 18, n. 3, pp. 301-307.

Ediciona; Dosdoce.com. "Digitalización del libro en España". Dosdoce. com, 2008.

http://www.dosdoce.com/continguts/estudios/contacto_cas.php?ID=22

eForo Publidisa. "Conclusiones". Publidisa, 2009.

http://www.publidisa.com/eforopublidisa/2009/conclusiones.asp

Federación de Gremios de Editores de España. Comercio interior del libro en España, 2006.

http://www.federacioneditores.org/0_Resources/Documentos/Comercio_ Interior_2006(R).pdf

Federación de Gremios de Editores de España. Comercio interior del libro en España, 2007.

http://www.federacioneditores.org/0_Resources/Documentos/Comercio_ Interior_2007.pdf

García-Marco, Francisco-Javier. "El libro electrónico y digital en la ecología informacional: avances y retos". El profesional de la información, 2008, julio-agosto, v. 17 , n. 4 , pp. 373-389. 
Harwood, Paul. "Courting controversy: views of a not-so-secret agent". Serials, 2003, march, v. 16, n. 1, pp. 97-98.

http://uksg.metapress.com/media/llbkvjttqm35k1mg9bt7/contributions/2/n/ $v / t / 2 n v t j k b 0 p v r d p q d b . p d f$

Larrañaga-Rubio, Julio. "El mercado del e-book en España: un análisis económico". Documentación de las ciencias de la información, 2008, v. 31, pp. 183-209.

Martín-González, Juan-Carlos; Pivetta, Emma. "Factores clave en el proceso de adquisición de libros electrónicos". El profesional de la información, 2008, julio-agosto, v. 17, n. 4, pp. 408-413.

Rodríguez, Joaquín. “Qué les preocupa a los editores?”. Weblogs $m i+d$. Los futuros del libro, 9 de octubre de 2007.

http://weblogs.madrimasd.org/futurosdellibro/archive/2007/10/09/75972.aspx

Teicher, Craig. "The customer is king at digital book 2008". Publishers weekly, 15 de mayo de 2008.
http://www.publishersweekly.com/article/CA6561155.html

Irene-Sofía Romero-Otero, Elea Giménez-Toledo. Centro de Ciencias Humanas y Sociales del Consejo Superior de de Investigaciones Científicas. Albasanz, 26-28. 28037 Madrid.

irenesofia.romero@cchs.csic.es

elea.gimenez@cchs.csic.es

Juan-Carlos Martín-González. Swets. Nàpols, 227 2 a planta, 08013 Barcelona.

jmartin@es.swets.com

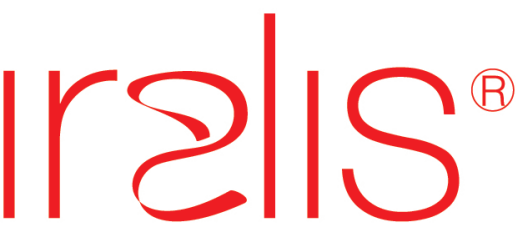

International Registry for Authors: Links to Identify Scientists

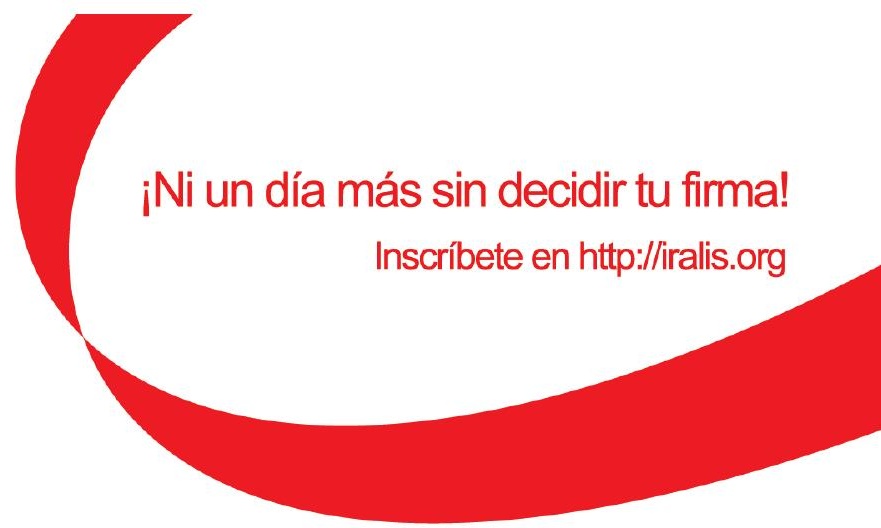

es:

- una guía para los autores hispanos para que firmen sus trabajos en el formato internacional usual

- una base de datos que registra las variantes de firma usadas por cada autor en diferentes épocas

- un buscador que usa automáticamente todas las variantes registradas

Con el apoyo de:

\section{El formato de ffrma internacional}

El profesional de la información 